\title{
Assessment of left ventricular cardiac shape by the use of volumetric curvature analysis from 3D echocardiography
}

\author{
Jeng-Ren Duann ${ }^{\mathrm{a}, *}$, Shan-Hui Chiang ${ }^{\mathrm{b}}$, Song-Bin Lin ${ }^{\mathrm{b}}$, Chung-Chih Lin ${ }^{\mathrm{c}}$, \\ Jyh-Horng Chen ${ }^{\mathrm{c}}$, Jenn-Lung $\mathrm{Su}^{\mathrm{b}}$ \\ ${ }^{\mathrm{a}}$ Institute of Applied Physics, Chung Yuan University, Chung Yuan University, Chungli, Taiwan 320 \\ ${ }^{\mathrm{b}}$ Department of Biomedical Engineering, Chung Yuan University, Chungli, Taiwan 320 \\ ${ }^{\mathrm{I}}$ Institute of Electric Engineering, National Taiwan University, Taiwan 106
}

Received 23 April 1998; accepted 10 November 1998

\begin{abstract}
A method for three-dimensional shape analysis of left ventricle (LV) is presented in this article. The method uses three-dimensional transesophageal echocardiography (TEE) as the source to derive the 3D wire-frame model and the related shape descriptors. The shape descriptors developed in this article include regional surface changing (RSC), global surface curvature (GSC), surface distance (SD), normalized surface distance (ND), and effective radius (ER) of the endocardial surface. Based on these shape descriptors, the shape of LV could be sketched in both static and dynamic manner. The results show that the new approach provides a robust but easy method to quantify regional and global LV shape from 2D and 3D echocardiograms. C) 1999 Elsevier Science Ltd. All rights reserved.
\end{abstract}

Keywords: Left ventricle; Cardiac shape analysis; 3D echocardiography; Surface curvature analysis

\section{Introduction}

Manipulation of loading condition, heart rate, contractility and myocardial perfusion by the use of physiological and pharmacological measures always influence the left ventricular volume and function [1-5]. This alteration of ventricular volume also affects the cardiac shape to some extent. Pathological conditions such as acute myocardial infarction or prolonged ischemic myocardium are often followed by the ventricular remodeling. It influences not only the cardiac shape and performance but also patient's prognosis. Deformation of left ventricular shape may also occur in the patient with left and right ventricular pressure and volume overloading [6-9].

Accordingly, the shape of heart is an important diagnostic and therapeutic index for evaluating a variety of cardiac diseases. Researchers have studied the relationship of the cardiac shape and the severity of heart diseases for several decades [10-18]. In the previous studies, the shape analysis methods were mainly based on two-dimensional tomographic section of the heart using simple indices or sophistical curvature analysis. In the study of Mancini et al. [14], a

* Corresponding author. Address: No 63, Kuo-Lien St.Long-Tarn, TaoYuan 325, Taiwan. Tel.: +886-3-456-3171/4558; fax: +886-3-480-1113.

E-mail address: djr@medical.be.cycu.edu.tw (J.-R. Duann) method was developed for measuring ventricular shape based on quantitative curvature analysis of ventricular outlines. The study was designed to assess prospectively the performance of the algorithm of quantitative curvature analysis and to compare it to traditional wall motion measurements. Their result showed that the shape analysis demonstrated a great concordance with the clinical diagnosis then did wall motion analysis. Besides, new information could also be provided by the quantitative curvature analysis, which needs no human involvement to decide the motion score of myocardial segments.

For quantitative analysis, the bending energy model was proposed by Duncan et al. [15] to measure the cardiac shape deformity. In this model, the left ventricle (LV) was simulated by an oval, which had some landmarks on it. The landmarks were also marked on the measured heart model with one-to-one correspondence to the landmarks on the oval model. The bending energy was derived from the distances of the landmarks on the measured heart model to that on the oval model. This evaluation could be used to indicate the deformity of the shape of heart.

The method of dynamic shape analysis proposed by Kass et al. [16] was used to evaluate the disease of aortic regurgitation and mitral regurgitation. The geometrical indices of eccentricity and circularity derived from the Fourier analysis were used to describe the changes of heart chambers. The 


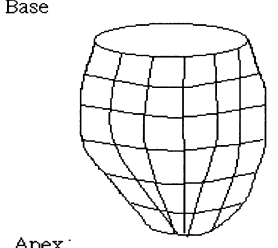

(a)

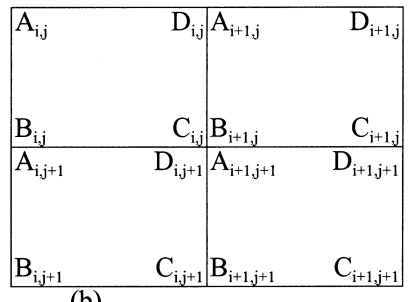

(b)
Fig. 1. The 3D wire frame model of the ventricle; (a) the 3D endocardial wire-frame model of the left ventricle; (b) four arbitrary wire meshes on the wire-frame model and their relative coordinates of mesh point that are used to derive the calculation of shape descriptors.

assessment revealed that the eccentricity derived from the patient with aortic regurgitation was higher than that of the normal during the ejection phase; however, the circularity obtained from the measurement of Fourier analysis was lower. In the measurement of the patient with mitral regurgitation, the chamber of the LV was lengthened during the systolic phase because of the returning blood. Most of these studies were very tedious to perform, and could not be represented real three-dimensional cardiac shape. Further, the results from two-dimensional shape analysis have often been over-extrapolated.

Several studies have shown that three-dimensional echo provide a better description of cardiac pathology and accuracy in quantification of ventricular volume and function than two-dimensional images [5,19-27]. In this article, we describe a new approach, the so-called, 3D volumetric curvature analysis (3DVCA) that yields the variety of shape descriptors on regional and global left ventricular shape from 3D echocardiographic images.

\section{Computation of three-dimensional shape descriptors}

The shape descriptors of regional surface changing (RSC), global surface curvature (GSC), surface distance (SD), normalized surface distance (ND), and effective radius (ER) of endocardial surface are developed and used to describe the shape of LV. Before measurement, the border of endocardial wall of $\mathrm{LV}$ is traced slice by slice. The area enclosed by the borders is cut into sectors with the same degree from the centroid to form a 3D wire-frame model of LV. The 3D shape descriptors are then derived from this 3D wire-frame model (Fig. 1). The following describes the definitions and calculations used to yield the shape descriptors of the left ventricular endocardial wall based on 3D wire-frame model.

\subsection{Regional surface changing}

The RSC describes the changing of the endocardial surface along the specified surface line of left ventricular endocardial wall. The following formulates the calculation of the RSC:

$\mathrm{RSC}=\sum_{i=1}^{M} \sum_{j=1}^{N}\left(\frac{\overrightarrow{a_{i+1, j} b_{i+1, j}}-\overrightarrow{a_{i, j} b_{i, j}}}{A_{i, j}}\right)$,

where $\overrightarrow{a_{i+1, j} b_{i+1, j}}$ is the tangent vector on the mesh point $(i+1, j), \overrightarrow{a_{i, j} b_{i, j}}$ is the tangent vector on the point $(i, j)$, and $A_{i, j}$ is the area surrounded by the mesh consists of vectors of $\overrightarrow{a_{i, j} b_{i, j}}$ and $\overrightarrow{a_{i, j} d_{i, j}}$. The indices $i$ and $j$ in Eq. (1) represent the range from mesh $[1,1]$ to mesh $(M, N)$ in which the endocaridal surface is taken into account. For example, if the range from $(0,0)$ to $(M, N)$ contains the area of the apex, the apical surface changing (ASC) is calculated. Similarly, if the range contains the area of septum, the septal surface changing (SSC) is evaluated.

The RSC accumulates all instantaneous changing of the tangent from the mesh point $(i, j)$ to the next mesh point $(i+$ $1, j)$ inside the evaluated range. When the regional endocardial surface changing of the whole mesh points are derived and displayed, the portion of the maximum change, that is, the steepest direction on the endocardial surface can be easily figured out [28]. Accordingly, RSC can be used to indicate the direction of movement of a roller bearing if you put it on the base of LV.

\subsection{Global surface curvature}

The GSC measures the summation of the normal vector on every mesh point of the 3D wire-frame model along a specified surface line. The calculation of the GSC is given as following:

$\mathrm{GSC}_{j}=\sum_{i=1}^{M} \overrightarrow{N_{i, j}}=\sum_{i=1}^{M} \overrightarrow{a_{i, j} b_{i, j}} \times \overrightarrow{a_{i, j} d_{i, j}}$,

where $\overrightarrow{N_{i, j}}$ is the normal vector on an arbitrary mesh point $(i, j), i=1,2, \ldots, M$ and $j=1,2, \ldots, N$ represented the meshes contains the whole endocardial surface of the LV, $\overrightarrow{a_{i, j} b_{i, j}}$ and $\overrightarrow{a_{i, j} d_{i, j}}$, as shown in Fig. 1, are two adjacent tangent vectors of two arms extended from mesh point $(i, j)$, and the symbol " $x$ " means the cross product of these two vectors. The GSC, which means the global curvature of a specified surface line, measures the symmetry of the left ventricular chamber to the center of cavity and the index of the deformation of $\mathrm{LV}$ along a specified surface line [28].

\subsection{Surface distance}

The SD shows the length of an arbitrary surface line from base to apex. It could be approximated by summing the edge segments of each mesh of 3D wire-frame model along the entire surface line. The following equation gives the calculation of SD:

$\mathrm{SD}_{j}=\sum_{i=1}^{M}\left|\overrightarrow{a_{i, j} b_{i, j}}\right|$ 
where $\mathrm{SD}_{j}$ is the $\mathrm{SD}$ along the $j$ th surface line, $i=1,2, \ldots, M$ represented the $i$ th slice, and $\overrightarrow{a_{i, j} b_{i, j}}$ is the tangent vector on the mesh point $(i, j)$ along the $j$ th surface line. It shows the distance of the pathway along an arbitrary surface line from base to apex on endocardial surface. This arbitrary surface line can be chosen from base down to apex with any possible path. The larger the SD is the more convex or concave from the long axis of LV the surface represents. The roughness of the surface may also enlarge the parameter of SD. However, when comparing SD obtained from end-diastolic and end-systolic phase with each other, the changes in SD can analogize to the contractility of LV.

\subsection{Normalized surface distance}

The ND is the normalized version of SD and measures the extent of convexity or concavity along the specified surface line but repels the influence of heart size. The following equation formulates the evaluation of ND:

$\mathrm{ND}_{j}=\frac{\sum_{i=1}^{M}\left|\overrightarrow{a_{i, j} b_{i, j}}\right|}{\left|\overrightarrow{a_{1, j} b_{M, j}}\right|}$,

where $\mathrm{ND}_{j}$ means the normalized distance of the $j$ th surface line, $i=1,2, \ldots, M$ is the number of the slices, $\left|\overrightarrow{a_{1, j} b_{M, j}}\right|$ is the length measured from the beginning mesh point (on the base) to the end point (on the apex) of the $j$ th surface line, and $\overrightarrow{a_{i, j} b_{i, j}} \mid$ is the length of the vector $\overrightarrow{a_{i, j} b_{i, j}}$. The ND, similar to the parameter of $\mathrm{SD}$, can also be used to measure the convexity or concavity of the surface on the specified surface line. As it is normalized with the straightforward (shortest) distance of the surface line, ND turns off the affect caused by the different size of LV. When we compare ND obtained in end-diastolic phase to that obtained in endsystolic phase, the ND describes the changes in shape as time evolving. As ND reduces to unity from arbitrary number, it shows the trend of degeneration from a curved surface to a plane surface. Applying to describe the shape of $\mathrm{LV}$, it represents the evolution of endocardial surface from sphere-like to cylinder-like shape.

\subsection{Effective radius of endocardial surface}

The ER measures the distance from every mesh point to the long axis of LV. The long axis of LV is defined as a straight line connecting the center of mitral valve and apex. The following gives the calculation of ER:

$\mathrm{ER}_{i, j}=\left|(x, y, z)_{i, j}-\left(x_{c}, y_{c}, z_{c}\right)_{i}\right|$

where $E R_{i, j}$ is the ER from the $j$ th mesh point of $i$ th slice, ( $x$, $y, z)_{i, j}$ is coordinate of the $j$ th mesh point on the $i$ th slice, and $\left(x_{c}, y_{c}, z_{c}\right)_{i}$ is coordinate of the point where the $i$ th slice intercepts the long-axis of LV. When the ERs of the entire endocardial surface of LV are calculated, they are displayed in three-dimension as a 3D effective radius map (ER map). Both the stereo landscape and the contour map can be illustrated to represent the global change of the curved endocardial surface of LV.

\section{Data acquisition and manipulation}

In order to obtain the shape descriptors of LV, 3D-image data set of LV should be acquired and undergoes image reconstruction to form a 3D wire-frame model. The procedures for estimating shape descriptors are image acquisition, volume reconstruction, border tracing and resampling, shape descriptors calculation, and graphic display. The following gives the details of each item.

\subsection{Image acquisition}

The echocardiographic images are acquired with HP Sonos 2500 ultrasound imager (Hewlett-Packard, USA) with TEE probe operated in 4D protocol. It acquires the sequential imaging planes using the rotational approach from a transesophageal transducer position. Images are acquired by starting with the transducer in the transverse position. Then a rotation is performed in our examinations, using $2^{\circ}-10^{\circ}$, and $3 \mathrm{D}$ data for the images are acquired at each position.

\subsection{Volume reconstruction}

As TEE imaging protocol acquires images using the rotational approach, the 3D-image data set must be converted to the cubic format for further processing. The volume reconstruction process transfers the raw data from polar coordinate $(r, \theta, z)$ to Cartesian coordinate $(x, y, z)$. The necessary interpolation should be introduced to calculate and fill the signal loss caused in the image acquisition scheme.

\subsection{Border tracing and resampling}

When the cubic data set is transferred, the transaxial planes of echo images are reformed and traced by manual tracing with assistance of semi-automated interpolation. The endocardial borders are obtained from base to apex on a slice by slice basis. The endocardial borders are then resampled by cutting the area enclosed by the border into sectors with the same degree from the centroid. By the use of each intersection point of the segments, 3D wire-frame model of endocardial surface was constructed.

\subsection{Shape descriptors estimation}

After the wire-frame models are reconstructed, the Cartesian coordinate of every mesh point is taken into calculation according to their definition to yield the shape parameters. 


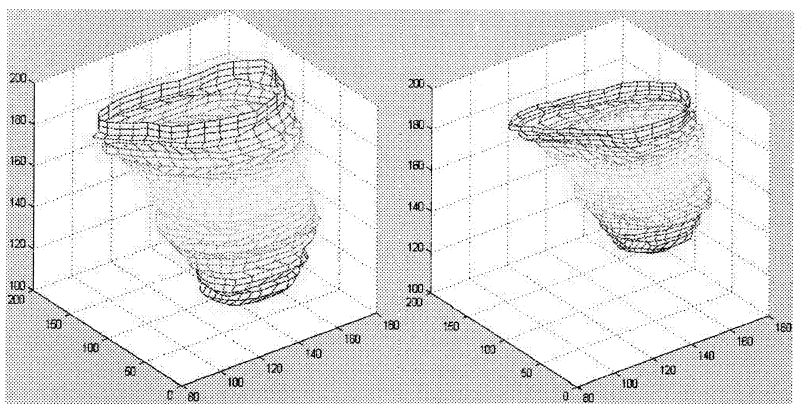

(a)

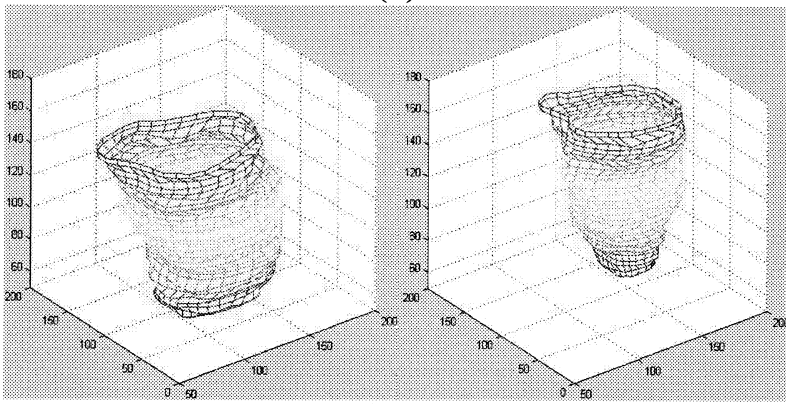

(c)

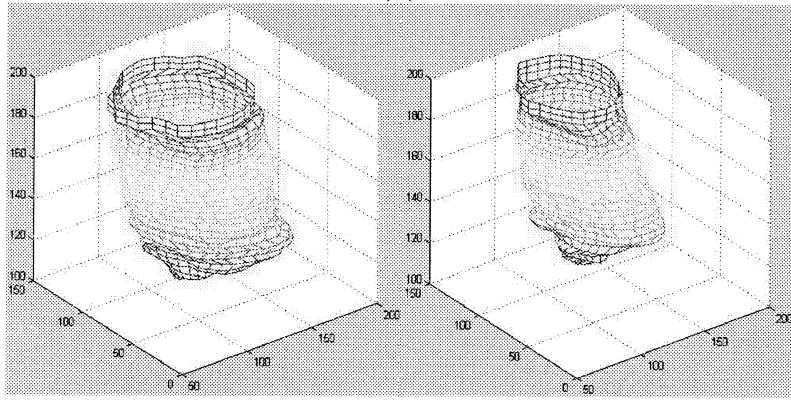

(e)

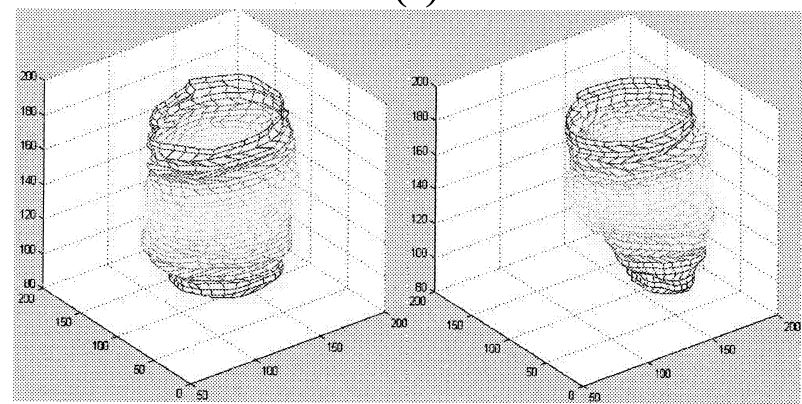

(g)

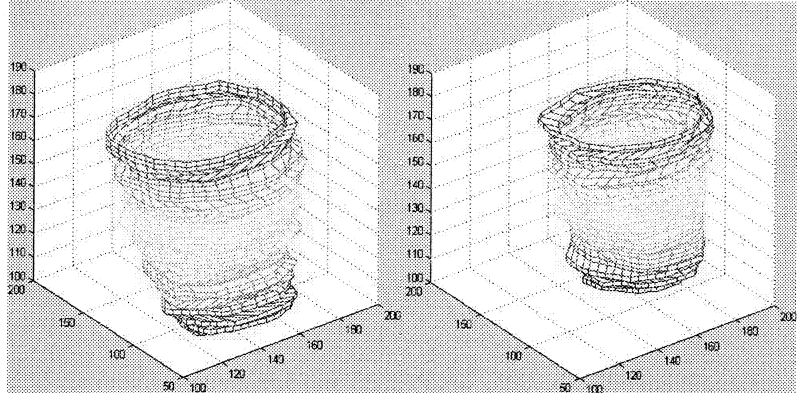

(b)

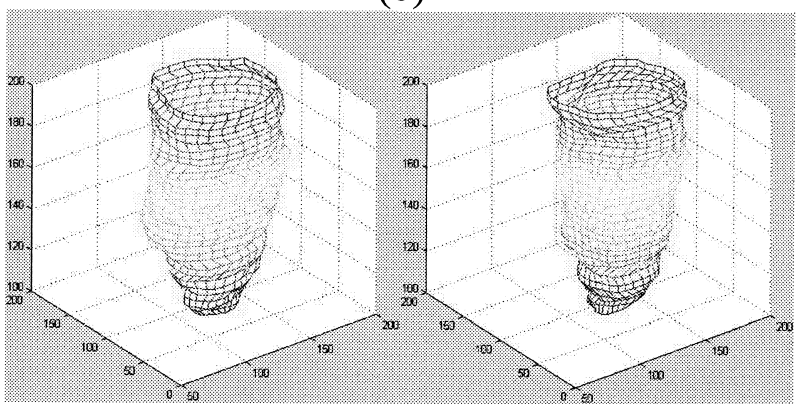

(d)

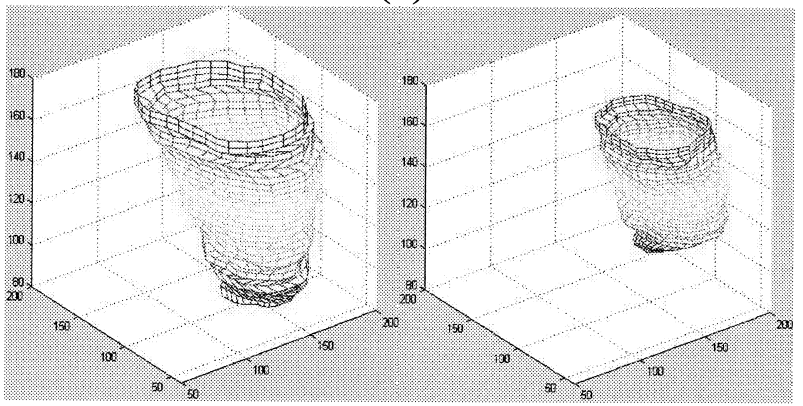

(f)

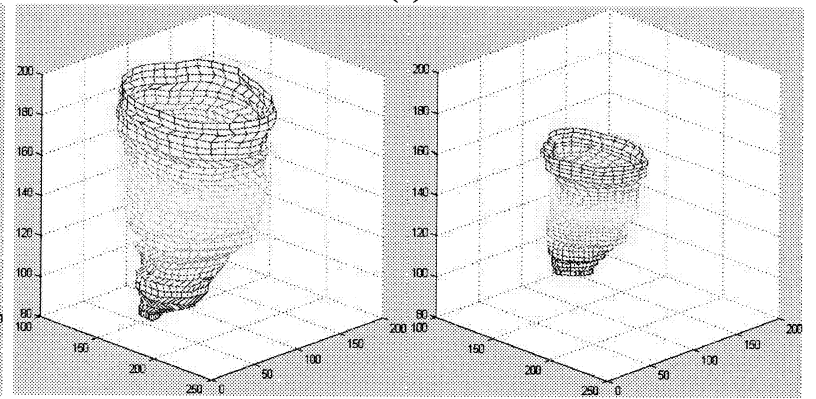

(h)

Fig. 2. Wire-frame models reconstructed from patients' left ventricle, from (a)-(h). The left panel shows the LV obtained in end-diastolic phase, the right panel shows that obtained in end-systolic phase.

\subsection{Graphic display}

The derived shape descriptors are displayed according to their characteristics. Generally, the 3D parameters such as ER and RSC are displayed in three-dimensional views. The others are displayed with two-dimensional curves or just calculated values.

The processes of volume reconstruction, border tracing and resampling, shape descriptor estimation, and graphic display are all custom-designed and performed on a personal computer. The volume reconstruction and border tracing and resampling processes are implemented with Microsoft Visual $\mathrm{C}++$ programming language. The parameter calculation and graphic display are developed under the Matlab environment. In despite of the edge tracing process, which is the most time-consuming procedure, the shape descriptor estimation processes could be completed within $1 \mathrm{~min}$. 


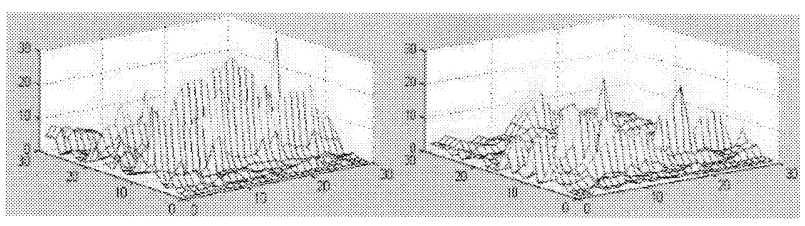

(a)

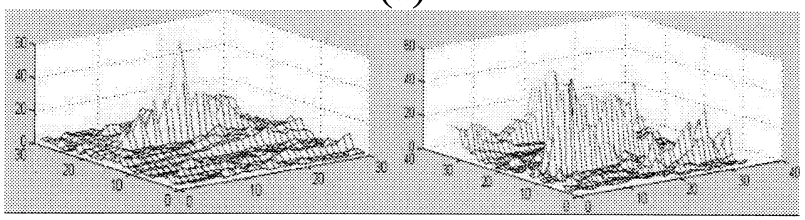

(b)

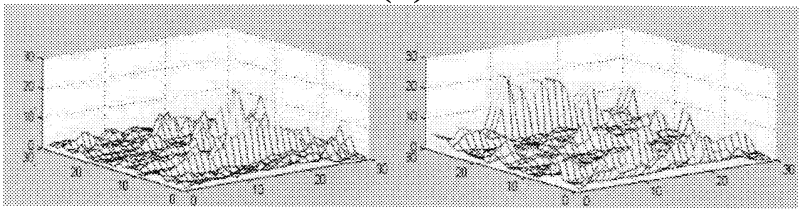

(c)

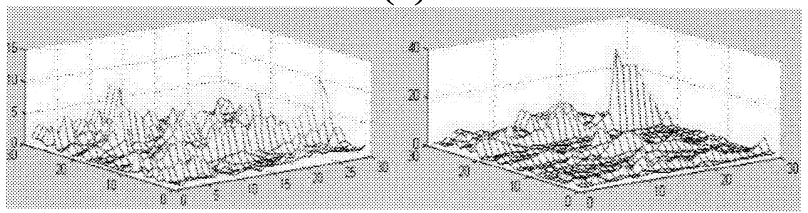

(d)

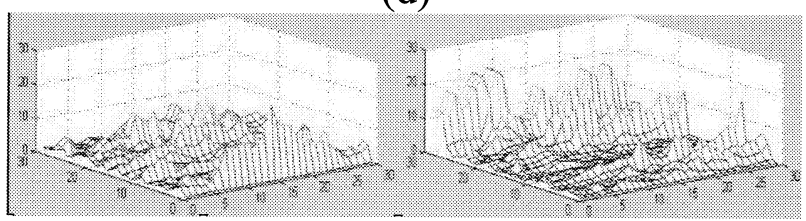

(e)

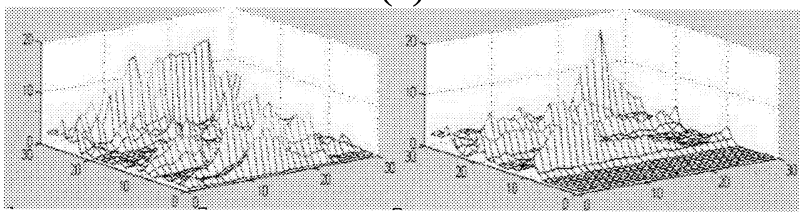

(f)

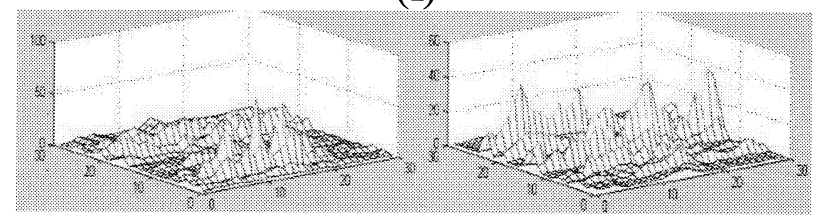

(g)

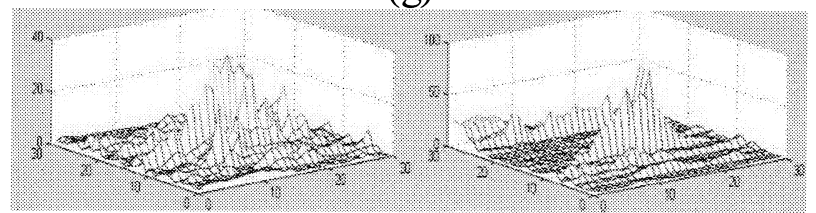

(h)

Fig. 3. The regional surface changing (RSC) of 3D wire-frame models obtained from eight patient's left ventricle. From (a)-(h), they show the results of patients $1-8$, respectively.

\section{System validations}

\subsection{Subjects}

Transesophageal echocardiography (TEE) of eight patients was acquired and underwent 3DVCA to verify the feasibility of the developed method. The 3D image data sets were acquired with HP Sonos 2500 ultrasound machine by TEE imaging probe operated under 4D protocol. After the cubic data sets were completed, the data sets in end-diastolic and end-systolic phases were chosen to perform 3DVCA to yield the shape descriptors. The shape descriptors obtained in both end-diastolic and end-systolic phases were compared.

\subsection{Tests of interobserver and intraobserver variations}

The variations of the interobserver and intraobserver were performed on one set of TEE images of phantom's (a balloon filled with known volume of water). The image data set underwent volumetric calculation and cardiac shape analysis to yield both the volume and the ER of the test phantom. All experiments were performed by two independent observers on separate occasions. Analysis of variation (ANOVA) of the volumetric calculation was computed with $F$-test with $p<0.05$. The variation of the ER was assessed by plotting the ER values obtained by one observer versus that obtained by himself or the other observer in one-to-one correspondence.

\section{Results}

\subsection{Measurements in echocardiographic images of patients}

A set of in vitro tests is performed with the TEE images of the patient's left ventricle. The reconstructed 3D wire-frame models of left ventricular endocardial surface obtained from the patients are shown in Fig. 2. Fig. 2a-h, show the 3D wire-frame models of eight patients' left ventricles in both end-diastolic and end-systolic phase. Fig. 3, shows the results of RSC obtained from the 3DVCA. The greater RSC means that the endocardial surface is rougher in this portion than elsewhere.

The GSC of the wire-frame models obtained from the patients' TEE images are shown in Fig. 4. Generally, the GSC of the end-diastolic phase is larger than that of endsystolic phase because of the fixation points at the end of apex and mitral annulus. In order to pour in more blood when diastole, the myocardium in the middle portion of LV must extend as much as possible. It enlarges the curvature of the left ventricular shape in the end-diastolic phase. The result also shows that the GSC is greater in free wall rather than the septum when measured in the end-diastolic phase. It means that the myocardial in free wall is more contractile and energetic than that in the septal area. From the result of Fig. 4, the change in GSC from end-diastolic 


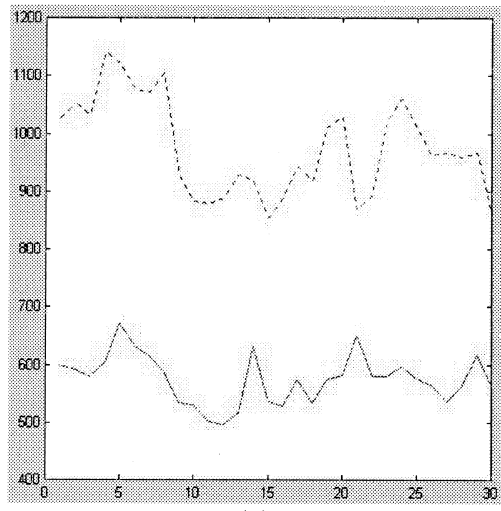

(a)

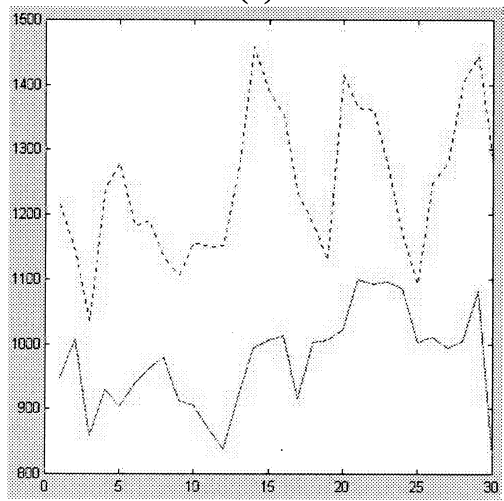

(c)

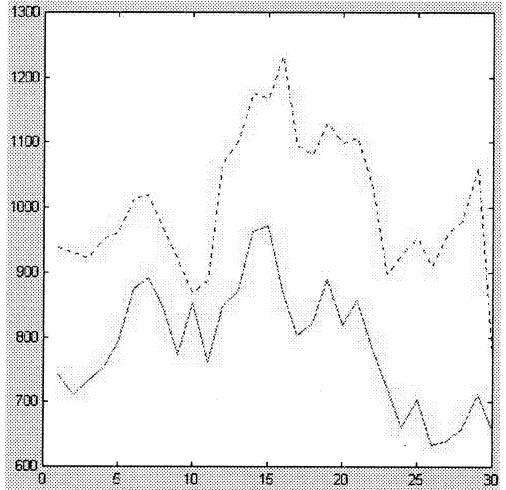

(e)

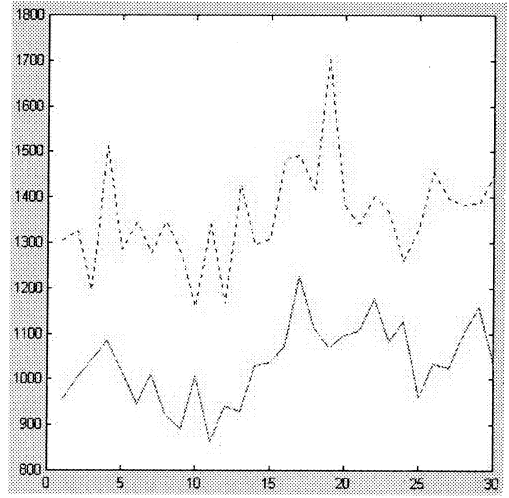

(g)

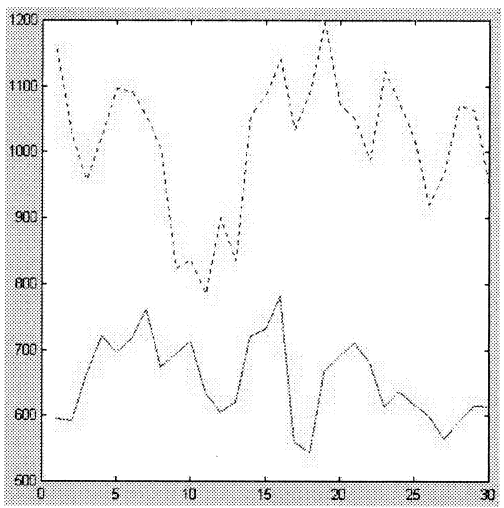

(b)

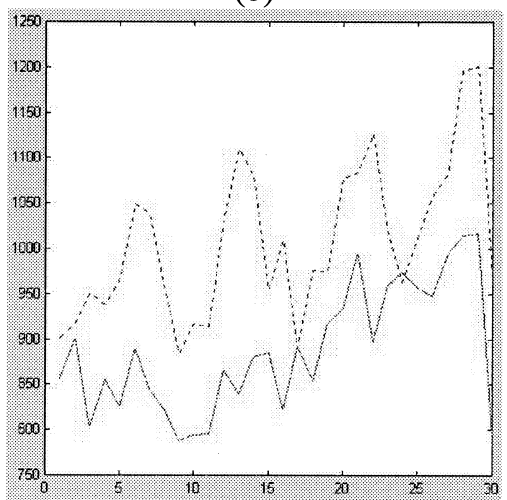

(d)

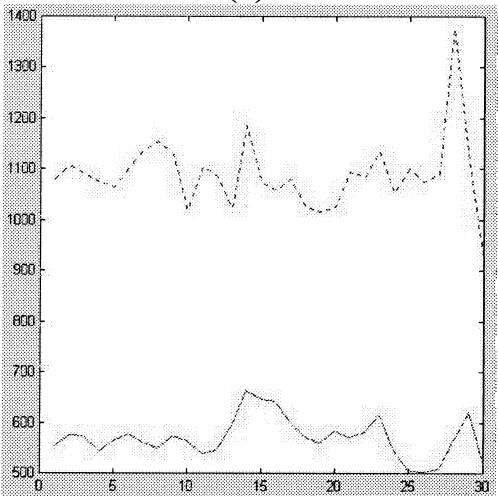

(f)

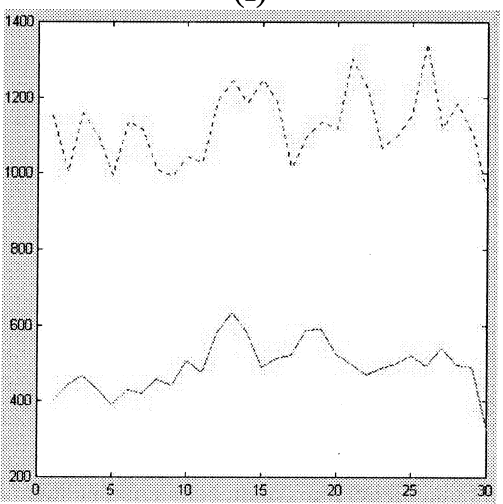

(h)

Fig. 4. The GSC obtained from the 3DVCA of eight patients' left ventricular TEE images. The dotted-line shows the result measured in end-diastolic phase and the solid-line shows the result obtained in end-systolic phase. 


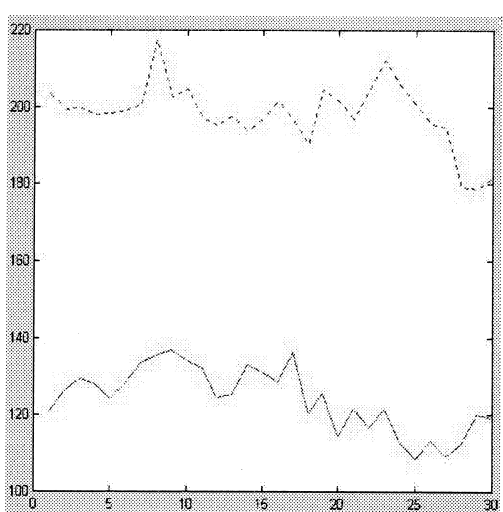

(a)

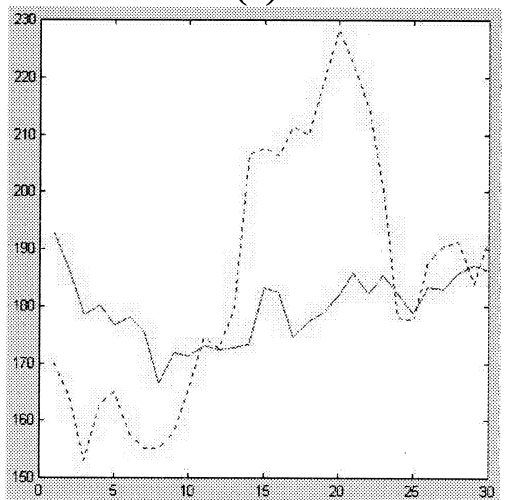

(c)

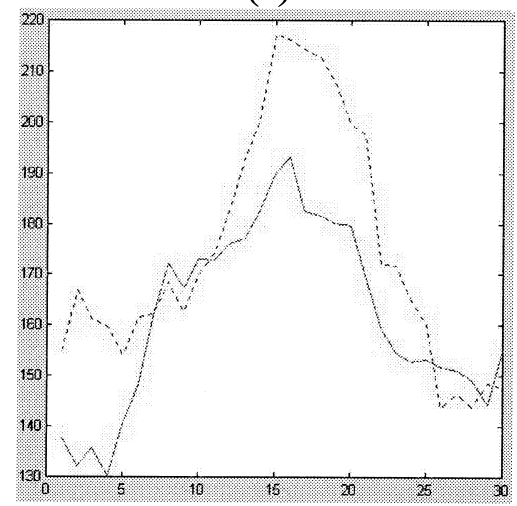

(e)

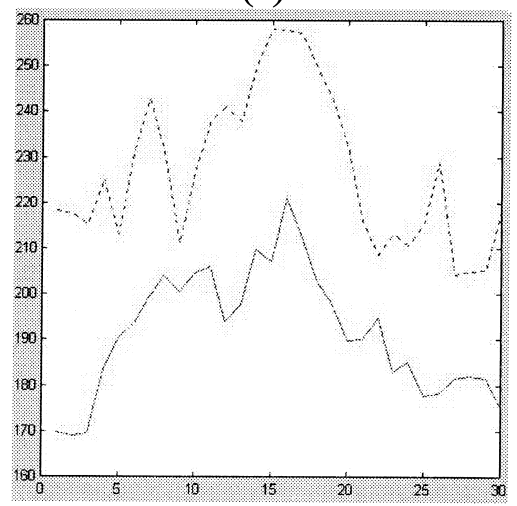

(g)

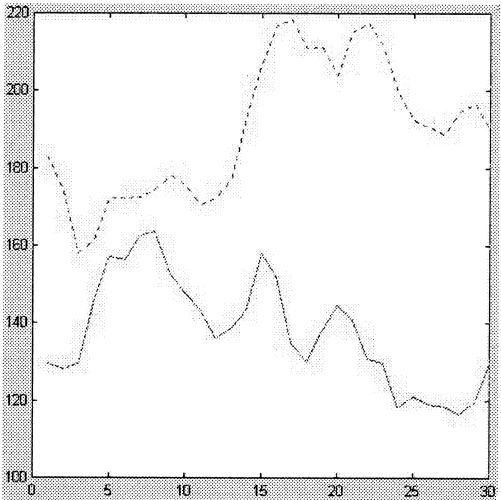

(b)

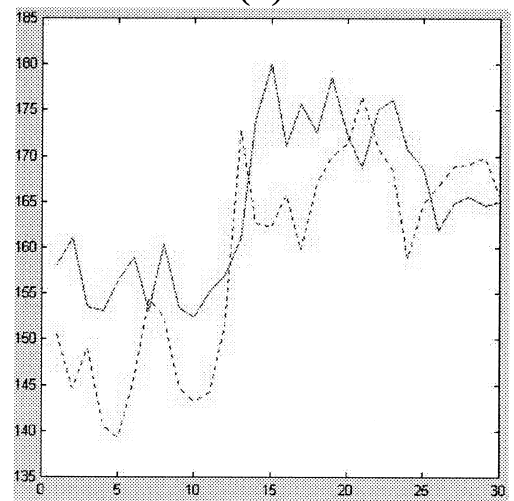

(d)

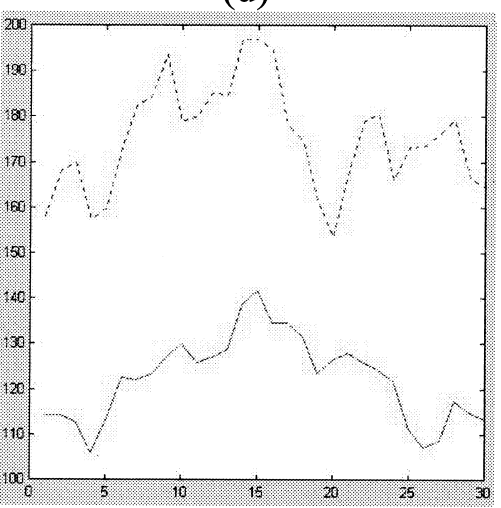

(f)

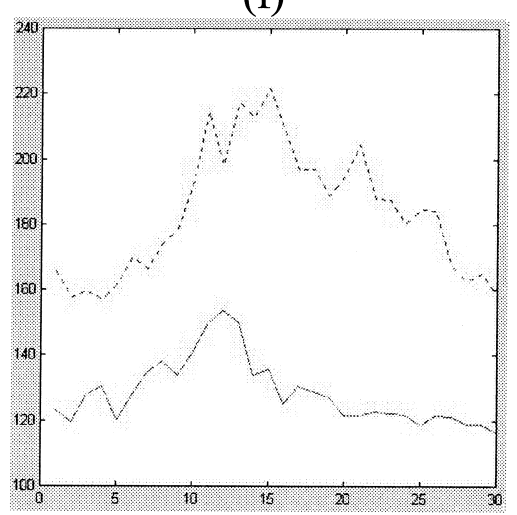

(h)

Fig. 5. The SD obtained from the 3DVCA of eight patients' left ventricular TEE images. The dotted-line shows the result measured in end-diastolic phase and the solid-line shows the result obtained in end-systolic phase. 


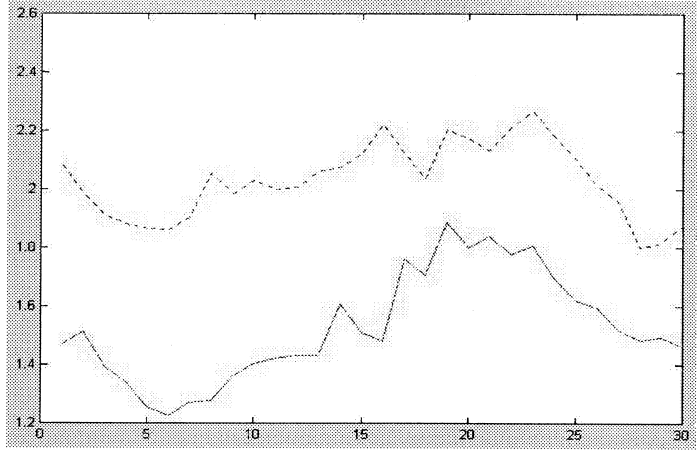

(a)

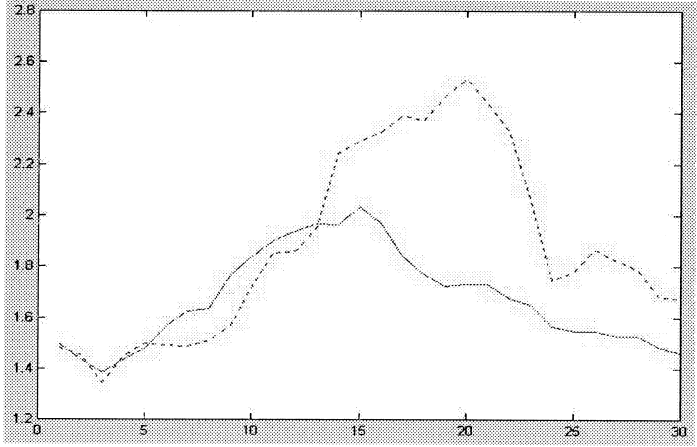

(c)

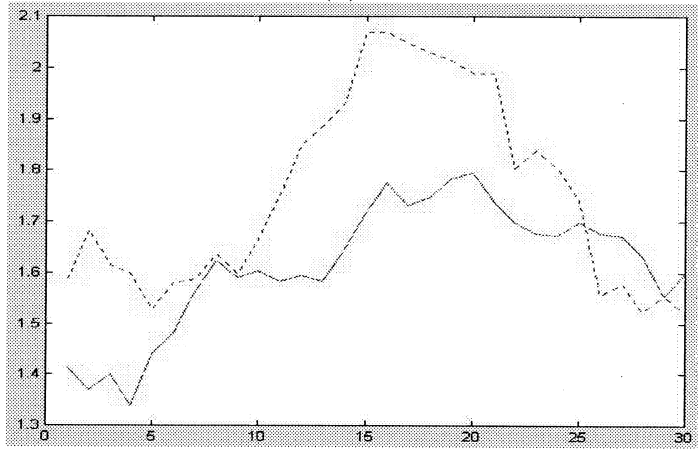

(e)

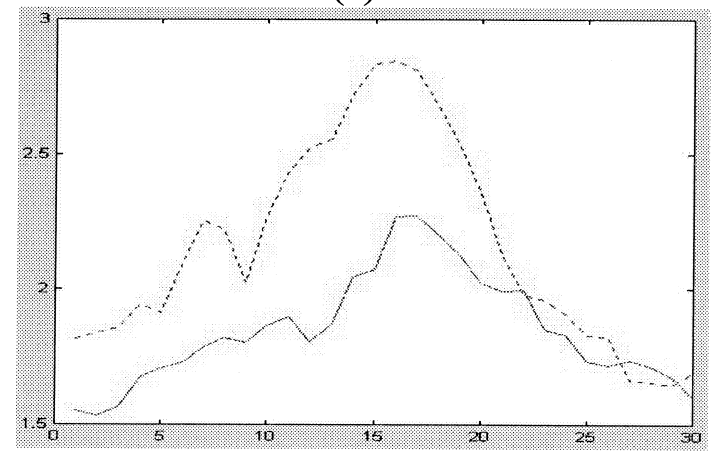

(g)

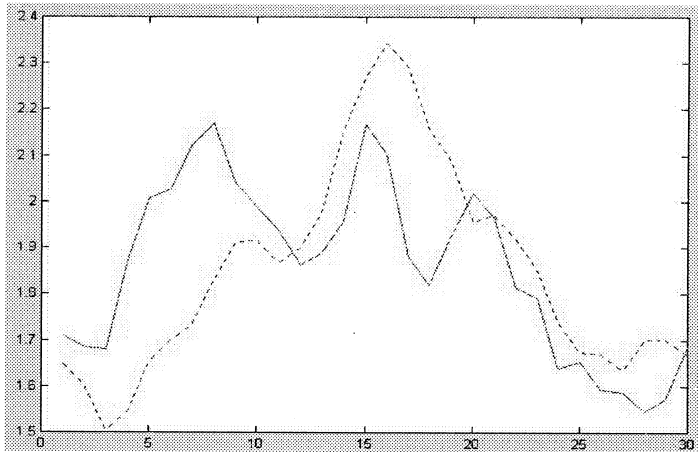

(b)

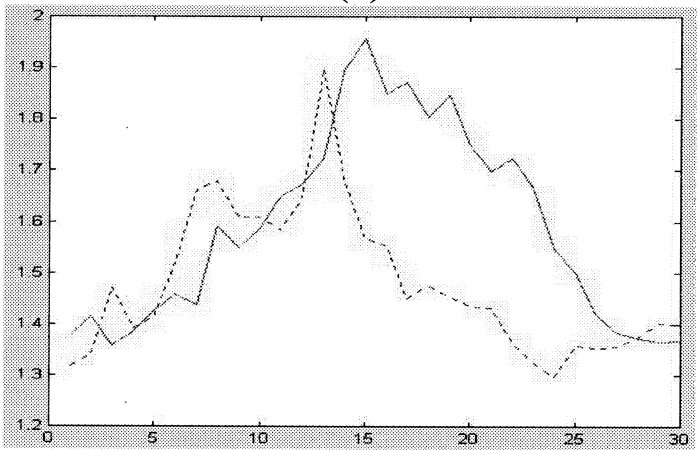

(d)

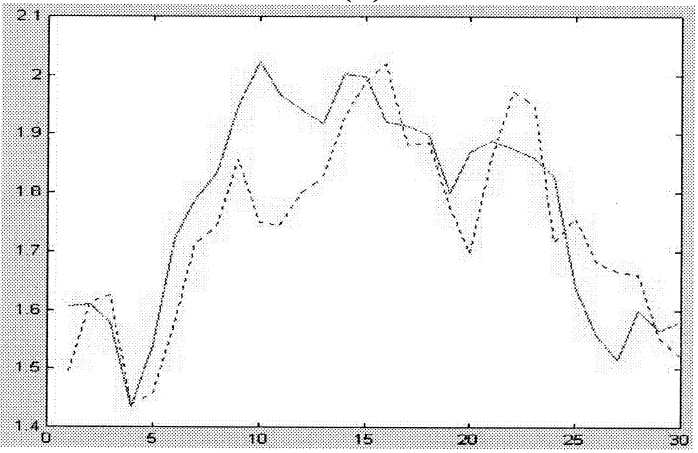

(f)

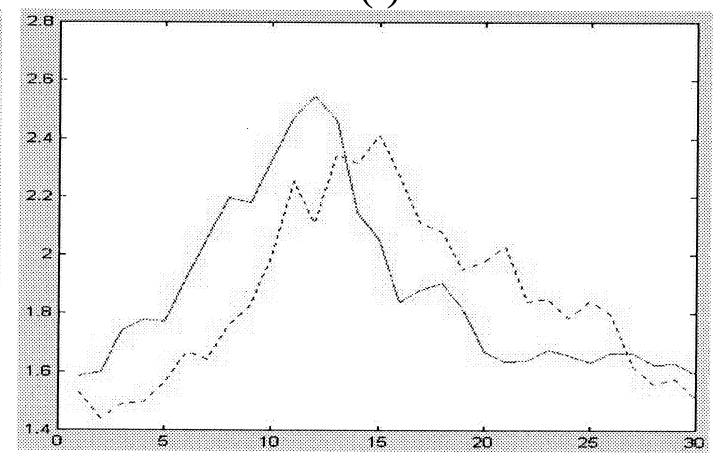

(h)

Fig. 6. The ND of eight patient's left ventricle, (a)-(h), obtained by the 3DVCA. In each figure, the dotted-line shows the ND obtained in end-diastolic phase, and the solid-line shows that obtained in end-systolic phase.

phase to end-systolic phase relates to the contractility of left ventricle. For example, the LV of patient 2 has a minor GSC change in the portion of LAD as shown in Fig. 4b; this might be caused by the ill contractile condition. However, the GSCs of patients 1 (Fig. 4a), 6 (Fig. 4f), and 8 (Fig. 4h) all change to a great extent because of the good contraction referred to in Fig. 2. The consistent waveform shown in Fig.4a, f, and h also 


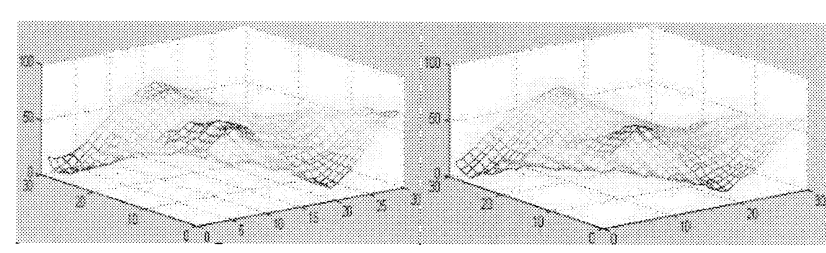

(a)

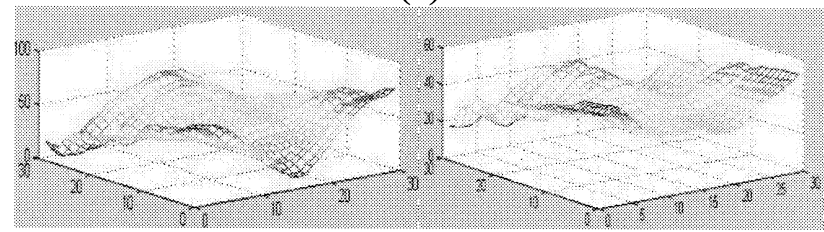

(b)

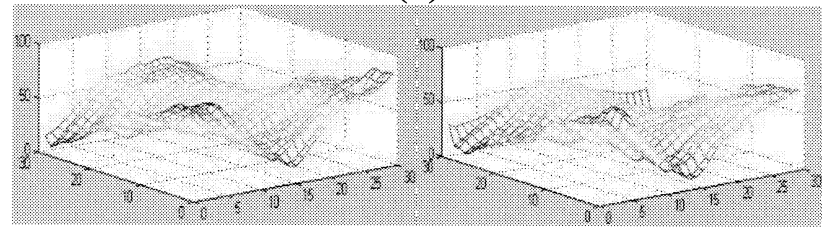

(c)

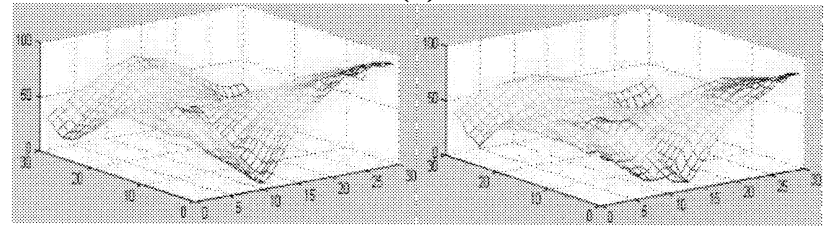

(d)

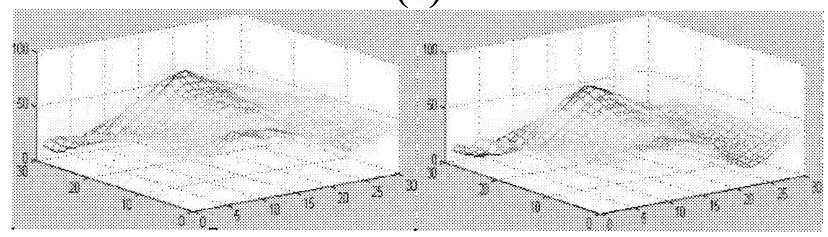

(e)

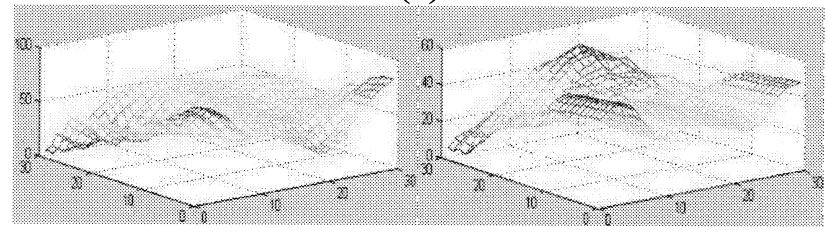

(f)

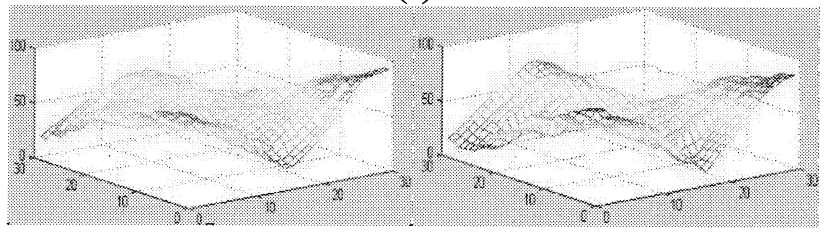

(g)

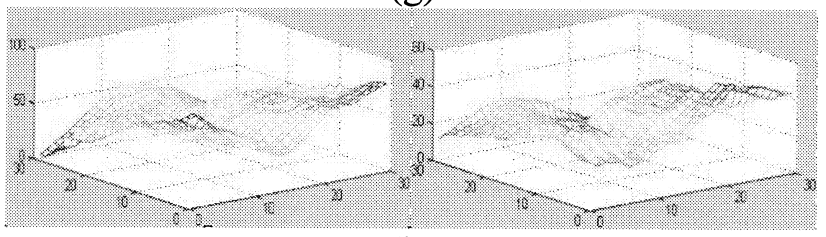

(h)

Fig. 7. ER map of endocardial surface by 3DVCA of eight patient's left ventricle. The figures in the left panel show the results obtained in enddiastolic phase and, correspondingly, the results obtained in end-systolic phase are shown in the right panel. indicates that the shape of endocardial surface is retained throughout the systole.

The SD that measures the length of a specified surface line from base to apex obtained from the patient's LV is depicted in Fig. 5. We can find that the area enclosed by the SD curve obtained respectively in end-diastolic and endsystolic phases is proportional to the contractility of myocardium. As the difference between the SD curve obtained in end-diastolic phase and that obtained in endsystolic phase in one-to-one correspondence represents the extent of contraction of the myocardium throughout the systole, it can also be considered as the index relates to the stroke work of heart. As the results show, patient 2 (Fig. 5b) had an ill contractile condition in the septal portion, the patients 3 and 5 had bad contractility in the portion of LAD as shown in Fig. $5 \mathrm{c}$ and e, the $\mathrm{LV}$ of patient 4 (Fig. 5d) had the worst contractility among all test patients.

Fig. 6 shows the results of ND obtained in end-diastolic phase (dotted-line) and end-systolic phase (solid-line). As the ND displays the trend of changes in shape of the endocardial surface throughout the systole, the difference between the NDs obtained in end-diastolic and end-systolic phase can be used to interpret the deformity of left ventricular chamber. For example, in Fig. 6a, it shows that the deformity of the entire LV is consistent throughout systole. Fig. 6c and d shows the deformity in free wall is stronger than that in septal portion.

The ER maps of eight patients' LV are displayed in Fig. 7. The left panel shows the ER maps obtained in end-diastolic phase and the right panel displays those obtained in endsystolic phase. Generally, the results show that the ER map obtained in end-diastolic phase is smoother than that obtained in end-systolic phase. This is because of the effect of upheaval of the injected blood in the end-diastolic phase.

\subsection{Interobserver and intraobserver variations}

The interobserver and intraobserver variations are assessed by two measurements of a set of ultrasonic images of phantom. One is the shape parameter of ER and the other is the volumetric calculation derived by summing all slice volumes. The relationships between the ER measured by the same observer and different observers are displayed by experiment or user basis (Fig. 8). Fig. 8a and b, displays the relationship between the measured ER of two measurements of observer 1 and observer 2 . The result shows that the variation of the measured ER obtained from observer 2 $(r=0.98)$ is slightly larger than the result obtained from observer $1(r=0.99)$. Fig. 8c, depicts the relationship between the measured ER obtained from observer 2 versus that obtained from observer 1 . The result shows that the correlation coefficient is 0.94 . That is, the measurement obtained by the different users is consistent with each other.

The assessment of the volumetric calculation is listed in Table 1. According to the result of variance analysis, the 
Effective Radius measured by the observer 1 at different occasions

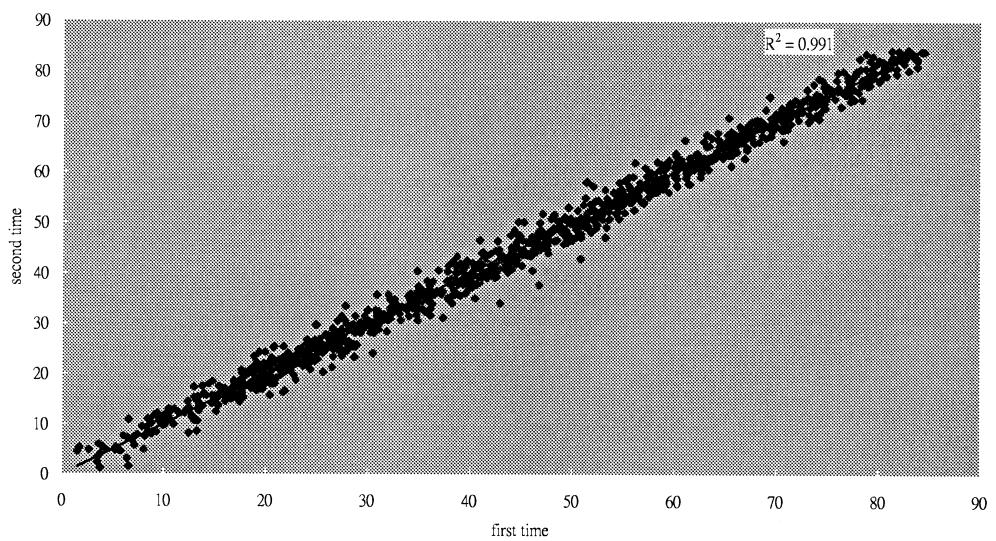

(a)

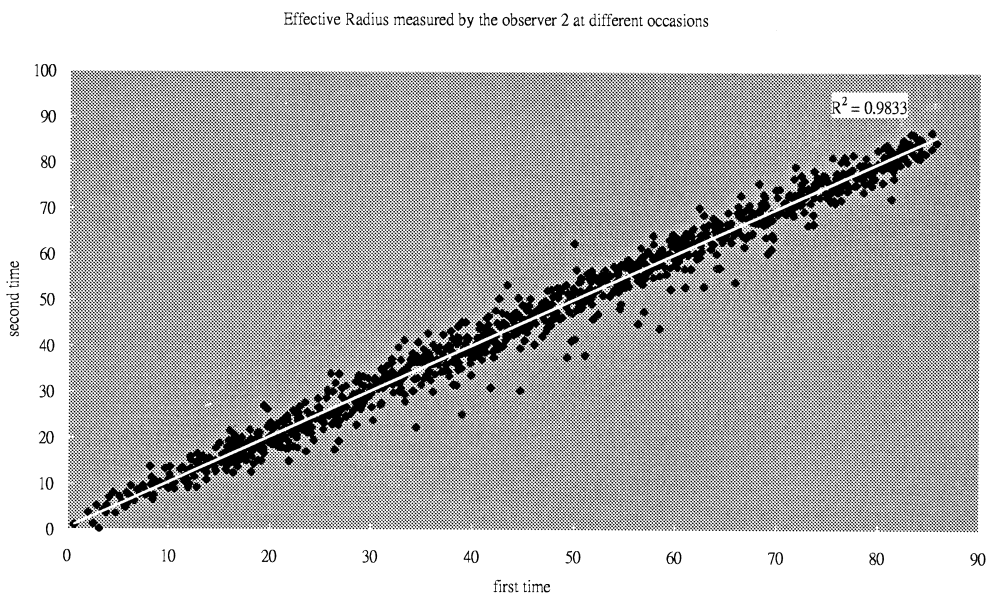

(b)

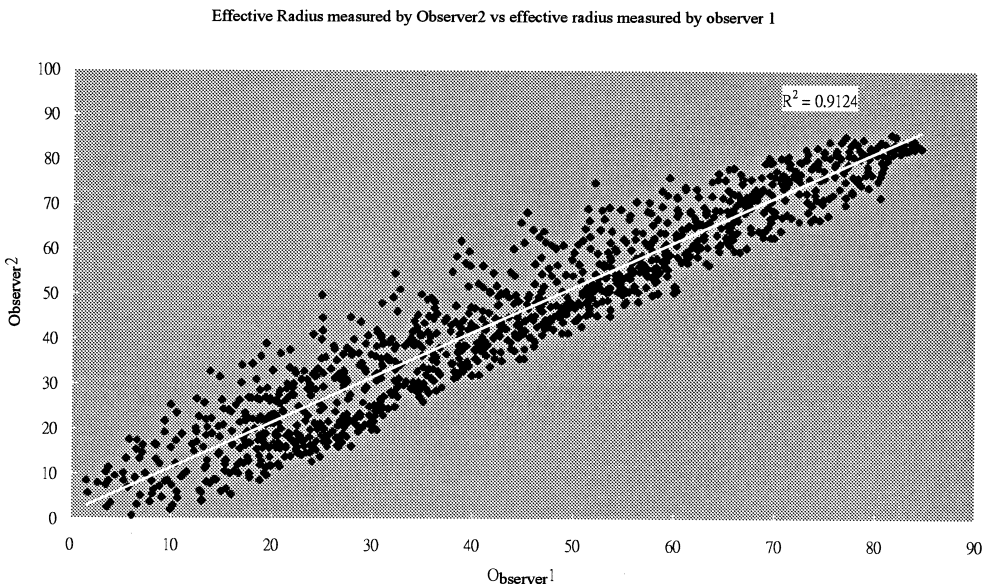

(c)

Fig. 8. The relationship between the measured ER obtained by the observer 1 and the observer 2, (a) the measured ER obtained by the observer 1 at two different occasions; (b) the measured ER obtained by the observer 2 at two different occasions; and (c) the measured ER obtained by the observer 2 versus that obtained by the observer 1 . 
Table 1

Variance analysis of the measurements of the volumetric calculation ${ }^{\mathrm{a}}$

\begin{tabular}{lcccc}
\hline & Observer 1 & Observer 2 & Row average & Row difference \\
\hline 1st measurement & 94.38757 & 97.75715 & 96.07236 & -0.80941 \\
2nd measurement & 95.28532 & 97.69740 & 96.49136 & -0.39040 \\
3rd measurement & 97.74256 & 98.46021 & 98.08158 & 1.19981 \\
Col average & 95.80515 & 97.95838 & Total average & 96.88177 \\
Col difference & -1.07662 & 1.07662 & & \\
\hline
\end{tabular}

${ }^{\text {a }}$ The $F$-value was 3.74 in rows and 4.83 in columns. Both differences in rows and columns were non-significant $(p<0.05)$.

$F$-value of the interobserver variation is 4.49 and, the $F$ value of the intraobserver is 4.83 . It indicates that both interobserver and intraobserver variations are non-significant during the volumetric calculation $(p<0.05)$. That is, the results of volumetric calculation are consistent with each other. The results show that the measurements are highly reliable and reproducible.

\section{Conclusion}

In this article, we described a set of shape descriptors allowing investigating 2D and 3D shape of $\mathrm{LV}$ regionally and globally. Custom-designed software to compute these shape descriptors was implemented and run on the Microsoft Windows environment. This was also validated with the echocardiograms of phantoms and patients. The results show that the developed shape descriptors can be applied to derive the multi-parametric data on the regional and global left ventricular shape. The software is also proved to be highly reliable and reproducible because of very low interobserver and intraobserver variability.

Despite that the 3D volumetric curvature (3DVCA) could be applied to explore the shape of the endocardial surface, we still need to analyze data from a large enough sample size to specify the relationship between the shape of LV and the cardiac diseases. In the near future, we are going to collect and categorize the three-dimensional image data set of echocardiographic images according to the cardiac disease and apply them to perform 3DVCA to identify the developed shape descriptors with their physiological and pathological implications. Besides, the 3DVCA should also be applied to the entire cardiac cycle rather than enddiastolic and end-systolic phases only to achieve the dynamic study of left ventricular shapes. However, the possible image data set obtained from other modalities, e.g., MR or computed tomography, should be applied to 3DVCA to be as cross-references for the current study.

\section{Summary}

Heart diseases often affect the left ventricular chamber size, myocardial mass and function. By the use of echocardiogram, we can reconstruct the 3D wire-frame model of LV and use it to derive various shape descriptors. The shape descriptors including RSC, GSC, SD, NSD, and ER of the endocardial surface could be derived from the three-dimensional wire-frame models generated from the 3D image data set.

The RSC could be used to measure the residual vector of moving direction. That is, it could tell the direction of roller bearing moving when it is placed on the top (base) of the endocardial surface. When the measured region contains either the apical or the ventricular septal portion of the heart, the apical (ASC) or septal surface changing (SSC) could be achieved. This parameter also indicates the extent of the roughness (or smoothness) of the surface around the apical or septal portion of LV. The GSC represents the symmetry to the center of mass of geometry. This could be used to indicate the degree of deformity of the heart dynamically. Additionally, the GSC also exhibits the relationship with contraction of endocardial surface. A normal LV shows consistent GSC waveform in both end-disatolic and end-systolic phase. The SD and its normalized version, ND, measure the length of a specified surface line. These two indices reveal the convexity and concavity of LV. Generally, the larger the SD or ND is, the more concave or convex the left ventricular endocardial surface appears. Besides, the comparison of the SD and ND obtained in both end-diastolic and end-systolic phases are made. The results depict that the change in SD with time evolution relates to the contractility and the stroke work of LV. However, the change in ND analogizes to the change in shape of endocardial surface with time evolving. The ER measures the distance from endocaridal surface to the geometrical long axis inside LV. However, ER could be displayed in threedimensional and would be useful to investigate the regional and global myocardial dynamics.

The developed descriptors are applied in the 3D cardiac shape of echocardiograms of phantoms and patients to validate the feasibility and physical meanings of them. The 3D wire-frame models are built with the procedures of image acquisition, cubic data reconstruction, edge tracing, and volume rendering. The shape descriptors are then evaluated from every mesh point on the 3D wire-frame models. The proposed method of three-dimensional cardiac shape analysis in this article could be applied not only in the description of the static and dynamic LV shapes, but also in evaluating other cardiac chamber and great vessel as well. These new parameters would be very useful in the study of 
pathophysiologic adaptation or mal-adaptation in a variety of cardiac diseases and would be profound clinical impact in the choice of appropriate therapeutic interventions and predicting patient's outcome.

\section{References}

[1] Wood AM, Hoffmann KR, Lipton MJ. Cardiac function: quantification with magnetic resonance and computed tomography. Card. Imag. 1994;32(3):553-579.

[2] Ali S, Egeblad H, Steensgard-Hansen F, Saunamaki K, Carstensen S, Haunso S. Echocardiographic assessment of regional and global left ventricular function: wall-motion scoring in parasternal and apical views versus apical views alone. Echocardiography 1997;14(4):313-320.

[3] Suzuki J, Caputo GR, Masui T, Chang JM, O’Sullivan M, Higgins CB. Assessment of right ventricular diastolic and systolic function in patients with dilated cardiomyopathy using cine magnetic resonance imaging. Am. Heart J. 1991;122:1035-1040.

[4] Lamb HJ, Doornbos J, van der Velde EA, Kruit MC, Reiber JHC, de Roos A. Echo planar MRI of the heart on a standard system: validation of measurements of left ventricular function and mass. J. Comput. Assist Tomogr. 1996;20(6):942-949.

[5] Belohlavek M, Foster SM, Kinnick RR, Greenleaf JF, Seward JB. Reference techniques for left ventricular measurement by threedimensional echocardiography: determination of precision, accuracy, and feasibility. Echocardiography 1997;14(4):329-335.

[6] Lessick J, Sideman S, Azhari H, Marcus M, Grenadier E, Beyar R. Regional three-dimensional geometry and function of left ventricles with fibrous aneurysms: a cine-computed tomography study. Circulation 1991;84:1078-1086.

[7] Yamaguchi S, Tamada Y, Miyawaki H, Niida Y, Fukui A, shirakabe M, Ohta I, Tsuiki K, Tomoike H. Resetting of regional preload because of ventricular shape change alters diastolic and systolic performance. Am. J. Physiol. 1993;265:H1629-H1637.

[8] Mancini GBJ, DeBoe SF, Anselmo E, Simon SB, LeFree MT, Vogel RA. Quantitative regional curvature analysis: an application of shape determination for the assessment of segmenttal left ventricular function in man. Am. Heart J. 1987;113(2):326-334.

[9] Hashimoto Y, Reid CL, Gardin JM. Left ventricular cavitary geometry and dynamic intracavitary left ventricular obstruction during dobutamine stress echocardiography. Am. J. Card. Imag. 1996;10(3):163-169.

[10] D'Cruz IA, Aboulatta H, Killam H, Bradley A, Hand RC. Quantitative two-dimensional echocardiographic assessment of left ventricular shape in ischemic heart disease. J. Clin. Ultrasound 1989;17:569572.

[11] Lever HM, Karam RF, Currie PJ, Healy BP. Hypertrophic cardiomyopathy in the elderly: distinctions from the young based on cardiac shape. Circulation 1989;79:580-589.

[12] Janicki JS, Weber KT, Gochman RF, Shroff S, Geheb FJ. Threedimensional myocardial and ventricular shape: a surface representation. Am. J. Physiol. 1981;241(10):H1-H11.

[13] Weiss JL, Eaton LW, Maughan WL, Brinker JA, Bulkley B, Guzman $\mathrm{P}$, Yin FCP. Ventricular size and shape by two-dimensional echocardiography. Federation Proc. 1981;40(7):2031-2036.

[14] Mancini GBJ, DeBoe SF, McFillem MJ, Bates ER. Quantitative regional curvature analysis: a prospective evaluation of ventricular shape and wall motion measurements. Am. Heart J. 1988;116(6):1616-1621.

[15] Duncan JS, Lee FA, Smeulder WMA, Zaret BL. A bending energy model for measurement of cardiac shape deformity. IEEE Trans. Med. Imag. 1991;10(3):307-320.

[16] Kass DA, Traill TA, Keating M, Altieri PI, Maughan WL. Abnormalities of dynamic ventricular shape change in patients with aortic and mitral valvular regurgitation: assessment by Fourier shape analysis and global geometric indexes. Circ. Res. 1988;62:127-138.

[17] Mancini GBJ, DeBoe SF, Anselno E, LeFree MT. A comparison of traditional wall motion assessment and quantitative shape analysis: a new method for characterizing left ventricular function in humans. Am. Heart J. 1987;114(5):1183-1191.

[18] Hutchins GM, Bulkley BH, Moore GW, Piasio MA, Lohr FT. Shape of the human cardiac ventricles. Am. J. Cardiol. 1978;41:646-654.

[19] Clarysse P, Friboulet D, Mafnin IE. Tracking geometrical descriptors on 3-D deformable surface: application to the left-ventricular surface of the heart. IEEE Trans. Med. Imag. 1997;16(4):392-404.

[20] Friboulet D, Magnin IE, Mathieu C, Pommert A, Hoehne KH. Assessment and visualization of the curvature of the left ventricle from $3 \mathrm{D}$ medical images. Computerized Med. Imag. Graphics 1993;17(4/ 5):257-262.

[21] McEachen JC II, Duncan JS. Shape-based tracking of left ventricular wall motion. IEEE Trans. Med. Imag. 1997;16(3):270-283.

[22] Maters BR, Senft SL. Transformation of a set of slices rotated on a common axis to a set of z-slices: application to three-dimensional visualization of the in vivo human lens. Computerized Med. Imag. Graphics 1997;21(3):145-151.

[23] Pai RG, Jintapakorn W, Tanimoto M, Cao QL, Pandian N, Shah PM. Three-dimensional echocardiography reconstruction of the left ventricle by a transesophageal tomographic technique: in vitro and in vivo validation of its volume measurement. Echocardiography 1996;13(6):613-621.

[24] Chen SY, Lin WC, Chen CT. Automated surface interpolation technique for 3-D object reconstruction from serial cross sections. Computerized Med. Imag. Graphics 1991;15(4):265-276.

[25] Jiang L, de Prada J, Handschumacher AV, Vuille MD, Guererro JL, Picard MH, Joziatis JT, Fallon JT, Weyman AE, Levine RA. Quantitative three-dimensional reconstruction of aneurysmal left ventricles: in vitro and in vivo validation. Circulation 1995;91(1):222-230.

[26] Gotteiner NL, Han G, Chandran KB, Vonesh MJ, Bresticker M, Greene R, Oba J, Kane BJ, Joob A, McPherson DD. In vivo assessment of nonlinear myocardial deformation using finite element analysis and three-dimensional echocardiographic reconstruction. Am. J. Card. Imag. 1995;9(3):185-194.

[27] Deng J, Gardener JE, Rodeck CH, Lees WR. Fetal echocardiography in three and four dimensions. Ultrasound Med. Biol. 1996;22(8):250291.

[28] Casey J. Exploring curvature. Braunchweig/Wiesbaden: Friedr. Vieweg and sohn Verlagsgesellschaft $\mathrm{mbH}$ 1996:250-291.

Jeng-Ren Duann received his B.S. and M.S. degrees in biomedical engineering from Chung Yuan University, Chungli, Taiwan in 1990 and $1992 \mathrm{He}$ is now the Ph.D candidate of the Institute of Applied Physics of Chung Yuan University. His current research interests include the construction of dynamic heart-beating model, medical signal and image processing, functional brain imaging, and medical informatics.

Shan-Hui Chiang received his B.S. degree in Biomedical Engineering from Chung Yuan University. She is now a graduate student of the Institute of Biomedical Engineering of Chung Yuan University. Her research interests include cardiac function, image processing and programming.

Song-Bin Lin received his BS degree in biomedical engineering from Chung Yuan University, Chungli, Taiwan in 1998. He is now a graduate student of the Institute of Biomedical Engineering of Chung Yuan University. His research interests are concentrated on the medical image processing and three-dimensional image reconstruction. $\mathrm{He}$ is now working on the development of automatic edge detection algorithm for echocardiography. 
Jenn-Lung Su received his B.S. (honours) degree in biomedical engineering from Chung Yuan University, Chungli, Taiwan in 1977. He received his M.S. and Ph.D degrees in bioengineering in 1985 and 1988, respectively, from The University of Illinois, Chicago. He was the Chairman of the Department of Biomedical Engineering, Chung Yuan University, from 1989 to 1995. Since 1988, he is an Associate Professor in Chung Yuan University. He also served as the Chief Editor of Chinese Journal of Medical and Biological Engineering since 1996. His current research interests include medical imaging, medical informatics, and electromagnetic interaction with biological systems.
Jyh-Horng Chen was born in 1960. He received his B.S. degree from Department of Electrical Engineering, National Taiwan University (NTUEE) in 1992. He then focused his interest on Biomedical Engineering for his M.S. degree from National Yang Ming Medical College and received his PhD degree in U.C. Berkeley/U.C. San Francisco Joint Bioengineering Program. He joined the faculty of NTUEE and is an associate professor since 1991. His research interests include medical informatics, magnetic resonance imaging, and man-machine interface for disables. Dr. Chen is a member of IEEE, SMRM and AAPM.

Chung-Chih Lin received his B.S. and M.S. degrees in biomedical engineering from Chung Yuan University in 1992 and 1994, respectively. He is a Ph.D scholar in the Department of Electrical Engineering, National Taiwan University (NTUEE). His research interests include medical informatics, WWW application, multimedia, database, and artificial neural network for biomedical application and data compression. 